\title{
Clinical, laboratory and radiological features predictive of survival outcome in severe COVID-19 in Wuhan, China
}

Jiaxing Xie ${ }^{1}$, yumei $\mathrm{Liu}^{2}$, qian Jiang ${ }^{3}$, cong Dong ${ }^{1}$, qin $\mathrm{Liu}^{4}$, jianjuan $\mathrm{Ma}^{5}$, xiaoxian Zhang $^{6}$, penghui $\mathrm{Wu}^{1}$, weihua Huang ${ }^{1}$, changxing $\mathrm{Ou}^{1}$, miaomiao $\mathrm{Hu}^{2}$, jianheng Zhang ${ }^{3}$, bomeng Zhang ${ }^{1}$, tingting Xia ${ }^{1}$, Lingling Cheng ${ }^{3}$, Xinlu Wang ${ }^{1}$, shiyue Li $^{3}$, qingsi Zeng ${ }^{1}$, qingling Zhang ${ }^{1}$, Kian Fan Chung ${ }^{7}$, haijun $\mathrm{Li}^{2}$, and Zhifang $\mathrm{Cai}^{8}$

${ }^{1}$ Guangzhou Institute of Respiratory Disease

${ }^{2}$ Hankou hospital of Wuhan City,

${ }^{3}$ State Key Laboratory of Respiratory Diseases, National Clinical Research Center for

Respiratory Disease, Guangzhou Institute of Respiratory Health, The First Affiliated

Hospital of Guangzhou Medical University

${ }^{4}$ The First Affiliated Hospital of Guangzhou Medical University

${ }^{5}$ Affiliated Hospital of Guizhou Medical University

6 State Key Laboratory of Respiratory Diseases, National Clinical Research Center for Respiratory Disease, Guangzhou Institute of Respiratory Health, The First Affiliated Hospital of Guangzhou Medical University

${ }^{7}$ Imperial College

${ }^{8}$ Hankou Hospital of Wuhan

May 15, 2020

\begin{abstract}
Objectives: We determined the clinical and imaging features of patients with severe COVID-19 that were associated with survival. Methods: Sixty-seven patients hospitalised with severe laboratory-confirmed COVID-19, were consecutively enrolled. Clinical data, blood measurements and chest computed tomographic (CT) scans were analyzed. Results: We compared the findings between 39 survivors and 28 non-survivors. At admission, although there were no differences in white blood cell (WBC) and platelet (PLT) counts, there was an increase of WBC, neutrophil, platelet distribution width and mean platelet volume with a marked decrease of lymphocyte, monocyte, eosinophil and PLT in non-survivor group on their last day compared to survivors $(\mathrm{P}<0.05)$. Non-survivors had higher ratios of peak creatinine $(\mathrm{P}<0.05)$ and peak lactate dehydrogenase $(\mathrm{LDH})(\mathrm{P}<0.05)$. Compared to survivors, the incremental rate of total lesion area, ground-glass opacity (GGO) area and consolidation area on $\mathrm{CT}$ scans was increased in non-survivors $(\mathrm{P}<0.05)$. The deceleration rate of total lung volume was greater in non-survivors than survivors $(\mathrm{P}<0.05)$. Using the univariate survival analysis, the following were predictive of non-survival: time from admission to peak of D-dimer $(\mathrm{D} 2 \mathrm{D})<16$ days, initial pro-BNP $>319.0 \mathrm{pg} / \mathrm{ml}$, peak procalcitonin $(\mathrm{PCT})[?] 0.19 \mathrm{ng} / \mathrm{ml}$, peak creatinine $>96.5$ $\mu \mathrm{mol} / \mathrm{l}$, peak alkaline phosphatase $(\mathrm{ALP})>81.5 \mathrm{u} / \mathrm{l}$, median time from admission to peak $\mathrm{ALP}<18$ days, the acceleration rate of total lesional area $>-11.5 \mathrm{~cm} 3 /$ day, incremental rate of GGO area $>2.4 \mathrm{~cm} 3 /$ day and the acceleration of consolidation area $>$ $2.3 \mathrm{~cm} 3$ /day. Conclusion: Hematological counts, serum analytes and radiological indicators, the latter assessed by artificial intelligence, are robust predictors of survival outcome in COVID-19.
\end{abstract}

\section{Hosted file}

combined version for Allergy-2020-5-14.pdf available at https://authorea.com/users/322364/ 
articles/451370-clinical-laboratory-and-radiological-features-predictive-of-survivaloutcome-in-severe-covid-19-in-wuhan-china 